\title{
A Unified Theory of Timing Budget Management
}

\author{
Soheil Ghiasi ${ }^{\dagger}$ \\ Elaheh Bozorgzadeh ${ }^{\ddagger}$ \\ $\uparrow$ Computer Science Department \\ University of California, Los Angeles \\ Los Angeles, CA 90095, USA \\ \{soheil,majid\}@cs.ucla.edu
}

\author{
Siddharth Choudhuri ${ }^{\ddagger}$ \\ \# Computer Science Department \\ University of California, Irvine \\ Irvine, CA 92697, USA \\ $\{$ eli, sid\}@ics.uci.edu
}

Majid Sarrafzadeh ${ }^{\dagger}$

\begin{abstract}
This paper presents a theoretical framework that optimally solves many open problems in time budgeting. Our approach unifies a large class of existing timemanagement paradigms. Examples include time budgeting for maximizing total weighted delay relaxation, minimizing the maximum relaxation and min-skew time budget distribution. We show that many of the time management problems can be transformed into a min-cost flow instance that can be optimally and efficiently solved through well-known combinatorial techniques. Experiments include mapping of several designs, which are implemented using parameterized CoreGen IP cores, on Xilinx FPGA devices. Different time budgeting policies have been applied during the mapping stage. Our time management techniques always improved the area requirement of the implemented testbenches compared to a widely-used path-based method. We also compared the maximum budgeting and fairness in delay budget assignments. Our experimental results show that an average improvement of $19 \%$ in area can be achieved when fairness and maximum budgeting policies are combined, compared to pure maximum budgeting.
\end{abstract}

\section{Introduction}

With tremendous growth in the complexity of today's systems, traditional design techniques are no longer capable of handling the design issues. One approach to tackle this problem is to design the system in a modular and hierarchical fashion, which requires the system level constraints to be translated into component level constraints. This task is generally referred to as budget management.

The problem of budget management has been studied for several design constraints including timing and area. Particularly, time budgeting is performed in order to slow down as many components as possible without violating the system's timing constraints. The slowed-down components can be further optimized to improve system's area, power dissipation, or other design quality metrics.

However, almost all of the previous research efforts employ sub-optima heuristics for addressing the reasonable formulations of the budgeting problem. In our previous work [11], we solved the problem of integral budget assignment optimally through LP relaxation. However, combinatorial methods are often preferred to LP-based approaches due to their numerical instability and slow runtimes.

In this paper, we present a unified theoretical framework that can solve different well-known formulations of the budgeting problem through efficient combinatorial techniques. We model the given application as a directed acyclic graph (DAG) and assign timing budget values to the edges of the DAG. Many other common budgeting models such as node budgeting or hybrid edge/node budgeting are special cases of our generic model. We show that our method optimally solves the problems of maximum, weighted, bounded, and fair budgeting. It also provides some guidelines for incremental budget re-assignment, which is useful for many practical applications.

Timing budget management is applied to different design tasks such as gate and wire sizing, and library mapping. To experiment our theoretical results, we apply our technique during the library mapping at datapath level. We integrate the time budgeting and module selection into the synthesis flow for mapping applications onto FPGA devices. Efficient time budgeting allows us to choose the proper modules from the library to obtain further quality improvements. Our results highlight that along with the total weighted summation of delay budget, its distribution throughout the design can significantly impact the design quality.
Next section presents some applications and related works. Section 3 formally formulates the problem. Section 4 presents a combinatorial optimal algorithm for solving the formulated problem. The problem is then extended to other budgeting policies in Section 5. We also show that all of the extensions are amenable to our approach. In Section 6, we discuss some interesting incremental properties of the problem, which are useful for incremental budget assignment. Some experiments supporting our theoretical results are reported in Section 7. Section 8 concludes the paper.

\subsection{Motivating Example}

Figure 1 illustrates an example of different delay budget assignment policies in action. All of the nodes in the example have unit intrinsic delay. Therefore, the critical path of the graph has length 4 . Delay budgets are assigned to the nodes and we assume that the timing constraint for the application is 8 time units. Hence, delay budget assignment should not create any path that takes longer than 8 units of time. For each cost function (policy), an optimal solution is depicted in the table. The delay budget assigned to each node is shown in each cell of the table. Note that the delay budget should be added to the unit intrinsic delay of the node to calculate its actual latency.

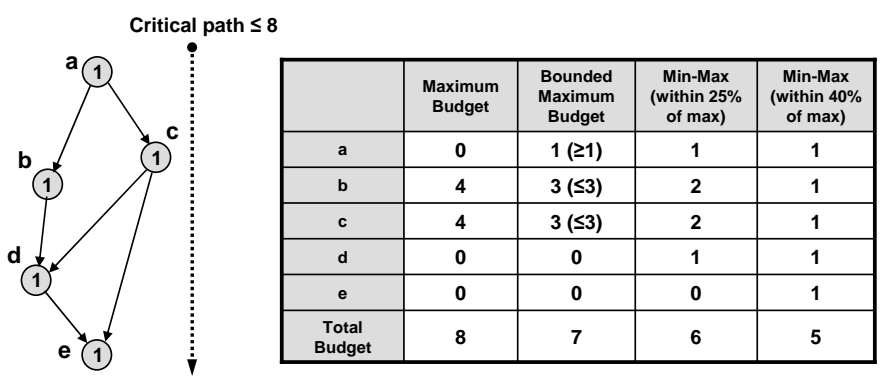

Fig. 1: Different timing budget management policies lead to different budget assignments. The example shows the optimal solution for maximum, bounded and min-max (with different total budgets) delay budget assignment. Our technique can accommodate all these policies optimally.

An intuitive budget assignment policy tries to maximize the total delay budget assigned to the graph, assuming that larger total budget correlates to larger improvements in the utility function. First column of the table shown in Figure 1 (maximum budgeting) represents the result of applying this cost function. A useful extension of this policy considers different weights for the nodes and tries to maximize the total weighted budget assignment.

Second column of the table (bounded maximum budgeting) illustrates the node budgets when the cost function tries to maximize the total budget while maintaining some lower/upper bounds on the amount of delay budget assigned to nodes. In this example, node $a$ has a lower bound of 1 , and nodes $b$ and $c$ have an upper bound of 3 on their delay budgets. Lower/upper bounds are useful for many application domains, due to non-linear relation of the utility function for each node to its delays.

Another popular policy is to distribute the budget values fairly (min- 
imizing the maximum budget value), while still trying to maximize the total budget. The last two columns of the table (min-max) represent the result of applying this policy to the sample graph, where $25 \%$ and $40 \%$ deviation from maximum budget is allowed for each case. Note that minimizing the maximum budget value has trivial solutions if there is no constraint on the total budget.

The technique that we present in this paper unifies all of the aforementioned budget assignment policies (plus a few others including minskew budget assignment) into one generic theoretical framework. We continue to present efficient combinatorial algorithms for solving all of the motivated problems.

\section{Related Works}

The concept of slack in a more general context has been addressed in the synthesis community, where negative slack for a node translates to the timing constraint violation. The budgeting problem on a graph (both temporal and spatial budgeting) has been studied in theory and practice and is widely used for many different applications.

- Design timing closure- During design optimization flow, timing budget is allocated to each node under a given timing constraint and optimization is applied. If timing constraint is not met, the delay budget is re-allocated $[4,20]$. Distribution of delay budget is applied to determine the wirelength under the given timing constraints.

- Timing-driven placement and floor planning- Delay budgeting during placement and floor planning has been extensively studied by several researchers $[1,3,16]$. In $[16,18]$, placement and net re-budgeting are combined. In [26], a novel technique for netweighting algorithm is proposed for timing optimization in placement. Recently, in [6], a new technique for delay budgeting on sequential circuits is proposed.

- Gate/wire sizing and power optimization- Under a given timing constraint, budget management can be applied to find a set of nodes/edges in the netlist graph such that their physical size or power dissipation can be reduced by mapping to smaller, or powerefficient cell instances with larger delays from a target library [3, $13,21]$

- Exploiting slack in high-level synthesis- There are several related work in the area of high-level synthesis where timing slack of the nodes in the data flow graphs are considered for better optimization in area and power. Examples are the algorithms and techniques developed for area minimization in pipelined datapath [24] and power minimization under timing constraint $[14,27]$.

- Layout compaction- Space budgeting has been studied in layout compaction in the field of VLSI CAD [28, 15, 12]. The concept of budgeting was first proposed by C.K.Wong et al. [28], for spatial budgeting in layout compaction.

Almost all of the techniques employed in these works are suboptimal heuristics such as Zero Slack Algorithm [23] and MISA [2]. In our previous work [11], we solved the problem of integral delay budgeting through LP relaxation. Due to the LP numerical instability and slow runtime, combinatorial methods are often preferred.

In this paper, we present an optimal combinatorial method for solving the integral budgeting problem. Furthermore, we utilize our method to solve the budgeting problem under many other objective functions, including weighted, bounded, and fair budgeting. Moreover, we present potentials for tackling the incremental budgeting problem. Our method is similar to the approach that Boros et. al have taken for the problem of balancing the data-flow graphs $[9,10]$. However, time budgeting problem is fairly different from their problem at hand. Moreover, to the best of our knowledge, this technique is quite novel in EDA community.

\section{Problem Statement}

Intuitively, the problem of timing budget management can be stated in the following way: Given an application with distinct constituting blocks, what is the maximum tolerable slow down of individual blocks without violating the timing constraints of the application? The sloweddown blocks can be further optimized to improve any of the generic design quality metrics depending on the application domain.

Although constituting blocks are often modelled as nodes in a DAG, the problem of delay budgeting for nodes is a special case of the more general edge budgeting case. This fact will be discussed in Section 5 in more details. Therefore, we focus on the edge budgeting problem. We assume that delay values and delay budgets are assigned to each edge of the DAG and nodes do not impose any delay on application paths. Edge budgeting has direct application in many areas such as routing and network optimization. Moreover, its special cases can solve node budgeting problem which applies to many other areas such as application and architecture synthesis.

Given an application represented by a DAG $G(V, E)$, there is a non negative delay value $d_{i j}$ and a non negative delay budget variable $b_{i j}$ associated to each edge $e_{i j} \in E$. There is a given value $T$ that specifies the timing constraint of the application. Timing constraint implies that all paths from any primary input to any primary output must take no longer than $T$. The delay of each path is calculated according to the following definition.

Definition: The delay of a path $p$ from node $s$ to node $t$ is equal to $\sum_{e_{i j} \in p}\left(d_{i j}+b_{i j}\right)$. We may use the terms delay of the path, cost of the path and the distance between nodes $s$ and $t$, interchangeably.

For simplicity, we add a virtual super input node $(S I)$ to $G$ that is only connected to all of the primary inputs. Similarly, there is a virtual super output node $(S O)$ that takes only all of the primary outputs of $G$ as its fanins (Figure 2). All of the edges connecting $S I$ or $S O$ to any other node in $G$ have zero delay. We still use $V$ and $E$ to represent the set of nodes and edges after adding $S I$ and $S O$ to $G$. The problem at hand can be formally formulated as maximizing $\sum_{e_{i j} \in E} b_{i j}$ such that all paths from $S I$ to $S O$ take no longer than $T$.

We assume that the problem input and output varibales are all nonnegative integers. This is particularly useful since for many application domains only discrete integer values are meaningful. Examples include high-level synthesis that deals with discrete clock cycles, or grid routing that can only handle discrete values for addressing grid coordinates.

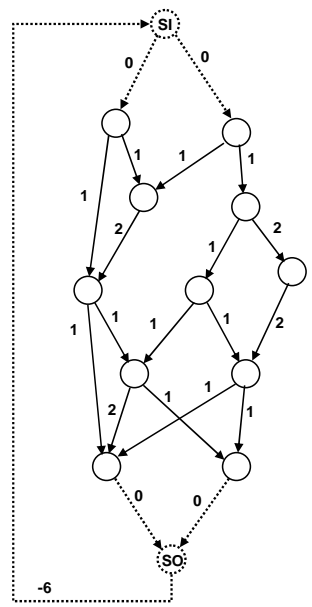

Fig. 2: A sample DAG with edge delay annotations. Nodes $S I$ and $S O$ and corresponding virtual edges are shown with dashed lines.

The problem can be stated as the following ILP formulation:

$$
\begin{array}{cc}
\operatorname{Max} \sum_{e_{i j} \in E} b_{i j} & \\
\sum\left(d_{i j}+b_{i j}\right) \leq T & \forall S I \rightarrow \text { SOpaths } \\
b_{i j}, d_{i j}, T \in Z_{+} & \forall e_{i j} \in E
\end{array}
$$

Directed acyclic graphs (DAGs) are usually utilized to model the applications at different levels of abstraction. Examples include task graphs modeling a high-level computation at the task level, data flow graphs 
representing applications at the architectural level and netlists modeling a gate level combinational circuit. The problem and techniques presented in this paper are valid on any DAG. Therefore, they are quite generic and applicable at different levels of abstraction.

\subsection{Equivalent Formulations}

The following lemma is a crucial observation that allows us to reformulate the problem.

Lemma 1. In an optimal budget assignment, the delay of all paths from SI to SO is $T$.

It follows that for a given graph $G$, the cost of a path from node $i$ to $S O$ does not depend on the choice of the path and is only a function of $i$. Let $r_{i}$ be a variable assigned to each node $i$ that represents its distance to $S O$. Therefore:

$$
r_{i}-r_{j}-d_{i j}=b_{i j} \quad \forall e_{i j} \in E
$$

Substituting this equation into the equations 1-3 leads to the following set of equations.

$$
\begin{gathered}
\operatorname{Max} \sum_{e_{i j} \in E} b_{i j} \\
r_{i}=r_{j}+d_{i j}+b_{i j} \quad \forall e_{i j} \in E \\
r_{S I}-r_{S O} \leq T \\
r_{i}, b_{i j} \in Z_{+} \quad \forall v_{i} \in V \text { and } e_{i j} \in E
\end{gathered}
$$

Note that the number of constraints in equations 1-3 can grow exponentially with respect to the number of nodes in the graph. However, formulation 5 has polynomial number of constraints with respect to the problem size. Moreover, we can assume that there is a virtual edge $e_{o i}$, from $S O$ to $S I$ with $-T$ delay (Figure 2). The constraint $r_{S I}-r_{S O} \leq T$ can be represented as one of the regular edge constraints and can be safely removed as a separate constraint. The example shown in Figure 2 assumes that the timing constraint for the application is 6 time units.

Utilizing equation 4 , we can eliminate $b_{i j}$ variables from the objective function by substituting $b_{i j}=r_{i}-r_{j}-d_{i j}$. Note that the non negativity constraint of $b_{i j}$ transforms to $\forall e_{i j} \in E: r_{i}-r_{j} \geq d_{i j}$. It follows that:

$$
\sum_{e_{i j} \in E} b_{i j}=\sum_{e_{i j} \in E} r_{i}-r_{j}-d_{i j}=\sum_{v_{i} \in V} r_{i}\left[\operatorname{out}\left(v_{i}\right)-i n\left(v_{i}\right)\right]-\sum_{e_{i j} \in E} d_{i j}
$$

a)
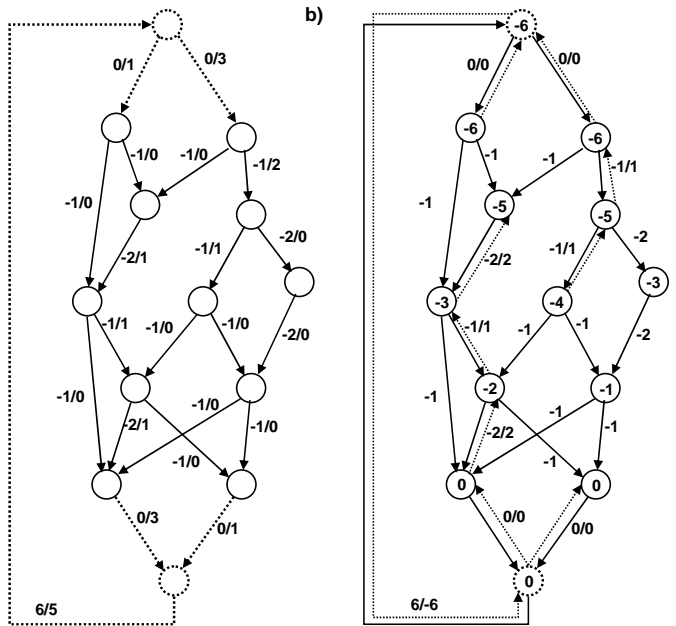

Fig. 3: a)The dual Min-cost flow problem/solution for example in Figure 2. Each edge is annotated with its cost and flow, respectively. Supply at each node $i$ is $i n\left(v_{i}\right)-o u t\left(v_{i}\right)$. b) The corresponding residual graph and edge costs. The number in each node shows the shortest path to SO $\left(\delta_{i}\right)$. where $\operatorname{in}\left(v_{i}\right)$ and $\operatorname{out}\left(v_{i}\right)$ are the in-degree and out-degree of vertex $v_{i}$, respectively. Note that the term $\sum_{e_{i j} \in E} d_{i j}$ is constant and can be eliminated from the objective function. Defining $\rho_{i}=\operatorname{out}\left(v_{i}\right)-\operatorname{in}\left(v_{i}\right)$, equations 5-8 can be rephrased as:

$$
\begin{aligned}
\operatorname{Max} \sum_{i \in V} \rho_{i} r_{i} & \\
r_{j}-r_{i} \leq-d_{i j} & \forall e_{i j} \in E \\
r_{i} \in Z_{+} & \forall v_{i} \in V
\end{aligned}
$$

the dual problem to the LP equations 9-11 is:

$$
\begin{gathered}
\operatorname{Min} \sum_{e_{i j} \in E}-d_{i j} y_{i j} \\
\sum_{e_{k i} \in E} y_{k i}-\sum_{e_{i j} \in E} y_{i j}=\rho_{i} \quad \forall v_{i} \in V \\
y_{i j} \in Z_{+} \quad \forall e_{i j} \in E
\end{gathered}
$$

Interestingly, the equations 12-14 formulate a conventional min-cost flow problem on the DAG, where $y_{i j}$ variables are the amount of flow along edges $e i j$ with cost $-d_{i j}$, and $\rho_{i}$ is the amount of demand at node $i$. Equivalently, $-\rho_{i}$ can be interpreted as the amount of flow supply at that node. Note that $\sum_{i \in V} \rho_{i}=0$ is satisfied as required in the min-cost flow problem $[22,9]$.

It follows that the original problem can be solved optimally in polynomial time. Section 4 presents an algorithm that can determine the value of $b_{i j}$ for each edge after solving the dual min-cost flow problem.

\section{Efficient Optimal Algorithm}

As shown in Subsection 3.1, the dual of the original edge delay budgeting problem can be stated as a min-cost flow problem on a new graph called $G^{\prime}(V, E) . G^{\prime}$ are $G$ are the same in terms of nodes and edges. However the cost of edge $e_{i j}$ is $-d_{i j}$ in $G^{\prime}$, and the amount of flow supply at node $v_{i}$ is $-\rho_{i}=\operatorname{in}\left(v_{i}\right)-\operatorname{out}\left(v_{i}\right)$. The flow supply has to be satisfied at each node by a feasible flow solution. Note that the cost of $e_{o i}$ is $T$. Hence, there is no negative cycle in the graph and the dual problem can be solved by any of the well-known min-cost flow algorithms [22]. Figure 3 illustrates the dual min-cost flow problem and its solution for the graph shown in Figure 2.

Once $y_{i j}$ variables (the amount of flow along edge $e_{i j}$ ) are figured out, we can construct the residual graph $G_{y}\left(V, E^{\prime}\right)$ from $G^{\prime}(V, E)$. For any edge $e_{i j}$ in $G^{\prime}$ with non-zero flow along it, there are two edges $e_{i j}$ and $e_{j i}$ in the residual graph. The cost of each backward edge $e_{j i}$ is $d_{i j}$, which is equal to the complement of the forward edge cost.

Let $\delta_{i}$ be the shortest distance of node $i$ to SO in the residual graph $G_{y}$. There is no negative cost cycles in the residual graph $G_{y}$ and hence, $\delta_{i}$ variables are well defined. $\delta_{i}$ variables can be determined by utilizing any well-known shortest path algorithm, such as Bellman-Ford algorithm [25], that is applicable to graphs with negative edge costs. Figure 3 shows the residual graph $\left(G_{y}\right)$ and $\delta_{i}$ variables for the example shown in Figure 3.

Variables $r_{i}$ and $b_{i j}$ of the primal problem can be easily calculated by substituting $r_{i}=-\delta_{i}$ and $b_{i j}=r_{i}-r_{j}-d_{i j}$. The following theorem proves that this simple equation determines the primal variables correctly.

Theorem 2. $r_{i}=-\delta_{i}$ is an optimal solution to the equations 9 , where $\delta_{i}$ is the shortest path of node i to $S O$ in the residual graph $G_{y}$

Proof: Corresponding to each flow variable $y_{i j}$ on edge $e_{i j}$, there is a constraint for that particular edge in the primal problem. According to the complementary slackness condition [8], we only need to assign values to $r_{i}$ variables such that the corresponding constraints become an equality for edges with non-zero flow. We now show that $r_{i}=-\delta_{i}$ satisfies this condition.

Suppose that $y_{i j}>0$ for an arbitrary edge $e_{i j}$ in $G^{\prime}$. Therefore, both edges $e_{i j}$ and $e_{j i}$ exist in $G_{y}$. Note that the cost of edges $e_{i j}$ and $e_{j i}$ are $-d_{i j}$ and $d_{i j}$ in $G_{y}$, respectively. According to the shortest path definition both of the following equations hold: 


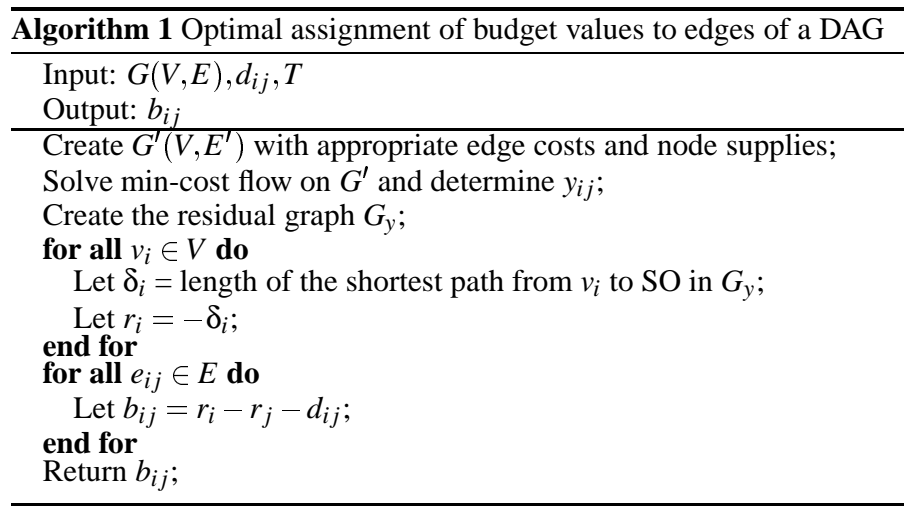

$$
\begin{aligned}
& \delta_{i} \leq \delta_{j}-d_{i j} \\
& \delta_{j} \leq \delta_{i}+d_{i j}
\end{aligned}
$$

Consequently, $r_{i}-r_{j}=\delta_{j}-\delta_{i}=d_{i j}$, which is exactly what complementary slackness condition implies.

Edge delay budgets are now easily calculated by $b_{i j}=r_{i}-r_{j}-d_{i j}$. This process is depicted in Algorithm 1. Well-studied techniques including min-cost flow and shortest path calculation are only used as a black box.

For the graph shown in Figure 2, the amount of the budget assigned to each edge of the graph is illustrates in Figure 4 utilizing Algorithm 1. Note that the number on each node is its distance to SO, and does not depend on the choice of path (Lemma 1). The amount of budget on edge $e_{i j}$ is readily given by $b_{i j}=r_{i}-r_{j}-d_{i j}$.

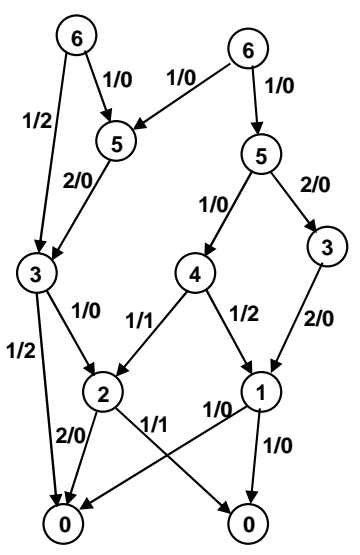

Fig. 4: Optimal budget assignment for the example in Figure 2. The numbers on each edge denote the delay and the assigned budget. Nodes are annotated with their delay to SO $\left(r_{i}\right)$.

\section{Extensions to Other Budgeting Policies}

The timing budget assigned to each of the design components can be exploited to improve different design quality metrics such as area, power dissipation, predictability or cost. The objective function presented in Section $3\left(\sum_{e_{i j} \in E} b_{i j}\right)$ assumes that a unit of delay budget assigned to any component will lead to the same amount of savings in the particular design quality metric of interest. This is not the case, however, in many practical situations.

For example, the amount of utility improvement per unit budget for a particular component might be twice as much of another component. Moreover, a component might be able to utilize only a limited amount of extra delay budget. Similarly, designers might need a minimum amount of delay budget assigned to some component in order to optimize it for some design metric.

In this section, we study the problem of delay budget assignment under various cost functions. We will show that many natural budget dis- tribution policies are simple extensions of what we presented in Section 3.

\subsection{Weighted Budget Distribution}

A unit delay budget can lead to different amounts of savings in the utility metric depending on the component that the budget is assigned to. In such cases, the budget assigned to different components of the application contributes to the cost function with different weights. Let non-negative $w_{i j}$ variables denote the weight of edge $e_{i j}$. The weighted budget assignment problem can be formulated as:

$$
\begin{array}{rc}
M a x & \sum_{e_{i j} \in E} w_{i j} b_{i j} \\
r_{i}=r_{j}+d_{i j}+b_{i j} & \forall e_{i j} \in E \\
r_{S I}-r_{S O} \leq T & \\
r_{i}, b_{i j} \in Z_{+} & \forall v_{i} \in \text { Vande }_{i j} \in E
\end{array}
$$

And similar to what we did in the previous section:

$$
\begin{gathered}
\sum_{e_{i j} \in E} w_{i j} b_{i j}=\sum_{e_{i j} \in E} w_{i j}\left(r_{i}-r_{j}-d_{i j}\right) \\
=\sum_{v_{i} \in V} r_{i}\left(\sum_{v_{j} \in \text { out }\left(v_{i}\right)} w_{i j}-\sum_{k \in \text { in }\left(v_{i}\right)} w_{k i}\right)-\sum_{e_{i j} \in E} w_{i j} d_{i j}
\end{gathered}
$$

which turns into the equations 9 after defining $\rho_{i}=\sum_{v_{j} \in \text { out }\left(v_{i}\right)} w_{i j}-$ $\sum_{k \in i n\left(v_{i}\right)} w_{k i}$. Therefore, the algorithm described in Section 4 can handle the weighted version as well. The only required modification is the demand function $\left(\rho_{i}\right)$ at each node for the dual min-cost flow problem.

An interesting special case of the weighted edge budgeting problem can solve the node budgeting problem, where each node has a delay value and we would like to assign budget values to nodes instead of edges. It is straight forward to see that a weighted node budgeting instance can be transformed into a weighted edge budgeting problem. The only required transformation is splitting each node into two other subnodes that are connected to each other by an edge with weight equal to the original node edge. All other regular edges should have zero weight. The technique is similar to the implementation of node capacities in a network flow instance that can handle only edge capacities. Note that some edges with zero weight might be assigned delay budgets in order to validate Lemma 1 .

\subsection{Bounded Budget Distribution}

The delay budget assigned to each component can be exploited to some specific extent. Extra budget assigned to a component would potentially be wasted, i.e., it will not lead to any utility improvement. In such cases, it is desirable to have a/an lower/upper bound on the delay of each edge. Budget lower bounds are easy to implement, since they can be added to the edge delay at the first place.

Upper bounds, however, are not as easy as the lower bounds to handle. In this subsection, we address the problem of maximum budget assignment under upper bound constraints on edges. We show that this problem boils down to solving the min-cost flow on a modified network and is similar to what we presented in Section 3.

Assume that there is an upper bound $u_{i j}$ for the delay of edge $e_{i j}$. Therefore, equations 9-11 change into:

$$
\begin{aligned}
\operatorname{Max} \sum_{i \in V} \rho_{i} r_{i} & \\
r_{j}-r_{i} \leq-d_{i j} & \forall e_{i j} \in E \\
r_{i}-r_{j} \leq u_{i j} & \forall e_{i j} \in E \\
r_{i} \in Z_{+} & \forall v_{i} \in V
\end{aligned}
$$

the dual problem to this problem is: 


$$
\begin{gathered}
\operatorname{Min} \sum_{e_{i j} \in E} u_{i j} z_{i j}-d_{i j} y_{i j} \\
\sum_{e_{k i} \in E}\left(y_{k i}-z_{k i}\right)-\sum_{e_{i j} \in E}\left(y_{i j}-z_{i j}\right)=\rho_{i} \quad \forall v_{i} \in V \\
y_{i j}, z_{i j} \in Z_{+} \quad \forall e_{i j} \in E
\end{gathered}
$$

Equations 25 formulate a min-cost flow problem on a network that is built by the following rule: For every edge $e_{i j}$ with cost $-d_{i j}$, there is a reverse edge $e_{j i}$ with cost $u_{i j} . y_{i j}$ and $z_{i j}$ are the amount of flow along edges $e_{i j}$ and $e_{j i}$, respectively. Note that by definition $d_{i j} \leq u_{i j}$. Hence, there is no negative cycle introduced into the network. Again, this problem can be solved using standard min-cost flow techniques. The solution to the primal problem will be easily determined after knowing $y_{i j}$ and $z_{i j}$ values.

\subsection{Min-Max Budget Distribution}

Fair distribution of the available budget to the application components is another reasonable objective. Fair distribution can be quantified by minimizing the maximum budget assigned to the graph edges, or minimizing the budget skew (the difference between the maximum and the minimum allotted budgets).

The problem of fair budgeting would have trivial ineffective solutions that minimize the maximum assigned budget or budget skew, if fairness was the only objective. For example, the zero budget assignment minimizes the maximum assigned budget. Similarly, one might increment the budget on each edge as long as the timing constraints are not violated, which leads to minimizing the budget skew. Therefore, fair budget distribution has to be considered as a supplementary objective to maximizing the total budget. For example, one might look for fair budget assignments among the solutions that have at least $\% 80$ of the total budget of an optimal solution.

In any case, the problem has to be initially solved using the algorithm described in Section 3.1 to maximize the total budget. Assuming that minimizing the maximum assigned budget (min-max) is the objective, we can perform binary search on the budget upper bounds to choose the best solution that has a reasonable total budget distribution. It follows that the algorithm complexity is only $O(\log (T))$ times more than that of the Algorithm 1. Similarly, we can search on edge lower and upper bounds at the same time to minimize the budget skew (the difference between the maximum and minimum assigned budget). However, this can increase the time complexity by a factor of $O\left(T^{2}\right)$ in the worst case.

\section{Potentials for Incremental Budgeting}

In many practical situations, the delay of a component and hence, the problem instance might change during the design flow iterations. Examples include, but are not limited to, library binding and physical design. Such problem instances can have millions of nodes in their representing graphs. Therefore, it is often impractical to re-execute the Algorithm 1 to find a new budget assignment for each local change. In such cases, it is often required to transform the current solution to a new feasible solution by performing local, rapid and incremental calculations.
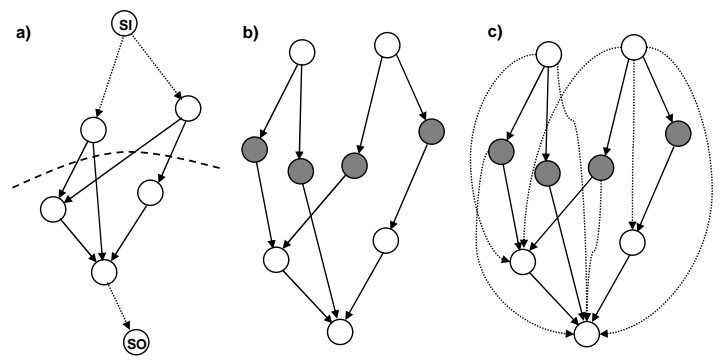

Fig. 5: a)A sample DAG and a sample cut are shown. b)The corresponding edge graph. The edges in the cut correspond to dark nodes. c) The transitive graph of the edge graph. The cut corresponds to the maximum independent set here.
In this section, we present some interesting properties of the problem formulated in Section 3. These properties can be further exploited to tackle other practical budgeting problems including incremental budget assignment. Due to the page limitation, some proofs or details of the proofs are eliminated.

Lemma 3. Flow variables of the dual LP problem will not change with the increase of the timing constraint $T$.

Proof: There is no negative cycle in $G^{\prime}$. Therefore, all paths that do not include $e_{o i}$ have costs not less than $-T$ (Figure 3 ). The min-cost flow algorithms work based on the augmenting path idea. In each iteration, a path with minimum cost from a source to a sink is found and the flow along that path is added to the solution. Therefore, increasing $T$ has no effect on the flows that run along paths that do not contain $e_{o i}$. Note that all such paths have negative cost.

However, edge $e_{o i}$ is the only backward edge of the graph $G$ and is common among the rest of the flows. Hence, increasing its cost will increase the cost for all such paths and will not change the choice of the path for min-cost flow algorithms. It follows that the flow variables will not change by increasing $T$.

Moreover it is easy to update the shortest path variables $\left(\delta_{i}\right)$ in the new residual graph. If the shortest path from a node to $S O$ passes through $e_{i o}$ in the original $G_{y}$, the value of the path is decreased by the amount of the increase in $T$. For other nodes, $\delta_{i}$ variables remain intact. It follow that if the timing constraint $(T)$ is increased by $\Delta_{T}$, the budget of a certain set of edges will be increased by $\Delta_{T}$. In other words we start from the original budget assignment that has been carried out under timing constraint $T$. $\Delta_{T}$ is then added to the budget of a particular set of edges.

Definitions: In graph $G(V, E)$, a subset of edges is called a cut if and only if every $S I$ to $S O$ path contains exactly one edge of the set (Figure 5). Graph $G^{*}\left(V^{*}, E^{*}\right)$ is called the intersection graph (or edge graph) of $G(V, E)$, if there is a node $v_{i j}^{*} \in V^{*}$ for every $e_{i j} \in$ $E$; and there is an edge $e_{i j k}^{*}$ between $v_{i j}^{*}$ and $v_{j k}^{*}$. Note that a cut in $G$ corresponds to an independent set of the transitive graph of $G^{*}$ $\left(G^{t *}\right)$. In the transitive graph, if there is an edge from node $v_{x}$ to $v_{y}$ and from $v_{y}$ to $v_{z}$, there is also an edge from $v_{x}$ to $v_{z}$. We use $G^{t}$ to represent the transitive graph of $G$.

Definitions: Let Gain $=O P T(G, T)$ denote the maximum amount of delay budget that can be added to graph $G$ under timing constraint $T$. Let Graph $G_{b}$ be the new graph that is formed by adding the delay budgets to the edges of $G$.

Lemma 4. For a given instance of the edge budgeting problem with critical path equal to $T$ :

Gain $=O P T\left(G, T+\Delta_{T}\right)=O P T(G, T)+O P T\left(G_{b}, \Delta_{T}\right)=O P T(G, T)+$ $\Delta_{T}\left|\operatorname{MIS}\left(G_{b}^{t *}\right)\right|=\operatorname{OPT}(G, T)+\Delta_{T}\left|\operatorname{MIS}\left(G^{t *}\right)\right|$

i.e. if the timing constraint $T$ is increased by $\Delta_{T}$, the budget of the edges that form a max-cut (a cut with the maximum cardinality) will be increased by $\Delta_{T}$. Such edges correspond to the maximum independent set in the transitive intersection graph of the problem instance and can be found using existing methods [7] (Figure 5). Therefore, incremental calculation of the budget assignment and edge delay budgets for various values of $T$ can be performed optimally.

The following lemma assists in performing budget re-assignment and incremental budget management.

Lemma 5. Let $G_{b}$ denote the graph after budget assignment. Let $c_{1}$ and $c_{2}$ represent two cuts in $G_{b}$, where all edges in $c_{1}$ are assigned at least $\delta$ units of budget. Decreasing the budget of all of the edges in $c_{1}$ by $\delta$, and increasing the budget of all of the edges in $c_{2}$ by $\delta$ leads to another maximal feasible solution (one that does not violate the timing constraint and all paths take exactly $T$ ).

\section{Delay Budget Assignment During Library Map- ping}

Delay budget assignment is applied during different design tasks at various stages of VLSI CAD flow. We apply delay budgeting during 
library mapping on a given datapath. We experimented with some applications from MediaBench [5]. We generated the dataflow graphs for these applications using SUIF compiler [19] and Machine-suif [17].

In Figure 6, the synthesis flow of mapping an application on an FPGA device is illustrated. This is a core-based implementation in which the computational operations are directly mapped to the existing optimized cores in the libraries. We use Xilinx VirtexE FPGA device as the target platform. Xilinx CoreGen tool is used to generate parameterizable cores optimized for the target architecture. For a given functionality, a number of cores can be generated to realize it with different characteristics such as latency. Figure 7 demonstrates the trade-off between the latency and the area in a 16-bit multiplier generated by CoreGen and mapped on FPGA VirtexE.

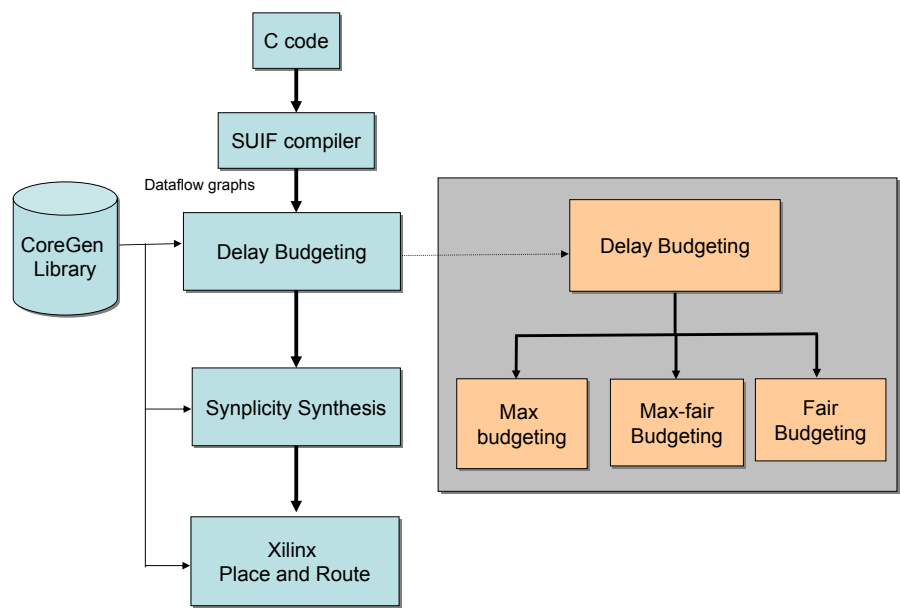

Fig. 6: Delay budgeting in core-based FPGA CAD flow.

From mediaBench applications, we selected the large dataflow graphs (DFGs) that could fit in the largest FPGA devices. The computations in the selected DFGs are multiplication, addition, subtraction, division, and shifting. We assume that all the data paths are 16-bit wide. Each computation is assigned to a resource generated from CoreGen tool based on delay budget allocated to the node.

The characteristics of the selected DFGs are outlined in Table 7. The latency column is the DFG latency plus additional slow-down for further delay budget injection. We apply different delay budgeting algorithms to allocate the delay budgets to the nodes (Figure 6). After library mapping and synthesis, the circuit is placed and routed on a FPGA device. We used the Synplify Pro 7.3 from Synplicity for synthesis and ISE 6.1 place and route tool provided by Xilinx.

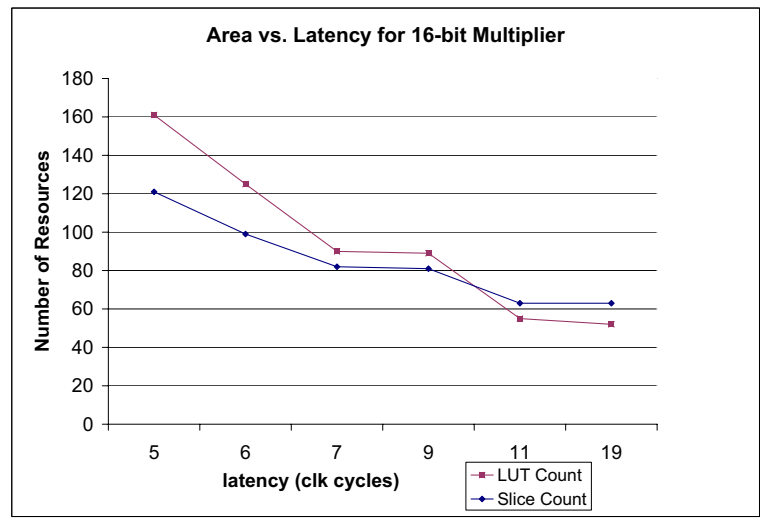

Fig. 7: Area vs. Latency for a 16-bit CoreGen Multiplier.

Figure 7 demonstrates almost linear trade-offs between the area and latency of the multiplier core in the library. Hence, maximizing the total budget can lead to further optimization in the area. In our previous work [11], we theoretically and empirically showed that optimal maximum budgeting outperforms ZSA [23] in reducing the design size. Maxi- mum budgeting reduced the design size by more than $10 \%$ on average compared to ZSA algorithm. In this work, we apply the min-max (fair) budgeting using the algorithm described in Subsection 5.3.

The objective function for this method is to maximize the minimum delay budget assigned to the nodes. In this technique, the total budgeting is compromised with the upper bound on the delay budgets. In the first set of experiments, we allow at most $10 \%$ degradation in the maximum total budget. In the second set, we allow more fair distribution of delay budget among the nodes by allowing at most $25 \%$ of degradation in total delay budget.

The results are outlined in Table 2. We compare different budgeting algorithms from two different perspectives: the objective value and the number of nodes with non-zero delay budgets (fair budgeting). Also, the impact of different techniques on total area is demonstrated. There are four different implementations for each DFG. In the first set called no_budgeting no delay budget assignment is applied and in the rest, different algorithms are applied for delay budget assignment.

\begin{tabular}{|c|c|c|c|c|}
\hline DFGs & Application & description & \# of nodes & Latency \\
\hline dfg0 & DSP & AR-lattice-filter & 18 & 20 \\
\hline dfg1 & mesa & matrix rotation & 28 & 17 \\
\hline dfg2 & mesa & invert matrix & 101 & 20 \\
\hline dfg3 & jpeg & idct & 58 & 23 \\
\hline
\end{tabular}

Table 1: Characteristics of DFG benchmarks.

The topology and connectivity in the applications affect the distribution of delay budgets in the graph. If most of the paths in the graph are critical, there is not much timing slack to be exploited. We assume that the timing constraint at the output of each application is its minimum possible latency plus some excess delay budget $(\Delta T=4)$. Therefore, more timing slack is injected to the graph before applying different delay budgeting algorithms.

The results on the area of the implemented designs (both LUT and slice count) show that the combination of fair and maximum budgeting can greatly reduce the design size. For most of the benchmarks, in the case of fair budgeting within $10 \%$ degradation in total budget the best results are obtained. Hence, the total injected budget, as well as the distribution of delay budgets both have a significant impact on the design quality. In $D F G O$ and $D F G 1$ the total budget does not change but fair distribution outperforms the other max budgeting with no fairness. For $D F G 2$, the fair budgeting with more degradation in total budget leads to better results. One reason is that the delay constraints for a larger number of nodes are relaxed (better distribution).

The last two columns compare the quality of different delay budgeting techniques. The column Variation in quality metric illustrates the percentage of variation in the quality metric (area) among different budgeting policies. The last column compares the best value of quality metric vs. the value where no budgeting technique is applied. The values in the last two columns are computed as the percentage of the difference between the best and the worst value in the set over the best: $\frac{\text { worst-best }}{\text { best }} \times 100$. On average, the area of designs after delay budgeting varies $18.08 \%$ in terms of LUT count (and $9.72 \%$ in terms of slice count) from the best depending on the algorithms used for budgeting. Applying delay budgeting improves the area by $53.66 \%$ (in terms of LUT count) and $35.83 \%$ (in terms of slice count) on average, compared to no budgeting.

\section{Conclusion}

We presented a theoretical framework that unifies a large class of existing time-management paradigms. Our model can optimally handle many time budgeting policies including maximizing total budget, weighted, bounded and fair budgeting. In addition, our technique is applicable to time management for edges, nodes and hybrid combination of these two elements.

We have performed experimental results on mapping some applications onto Xilinx FPGAs. We have generated the applications' datapath components using CoreGen IP cores. We have compared different time budgeting policies in terms of the design area under equal timing constraints. Experimental results exhibit significant savings in design quality (area in our experiments) and advocate our theoretical results. 


\begin{tabular}{|c|c|c|c|c|c|c|c|}
\hline \multirow[t]{2}{*}{ Benchmark } & \multirow[t]{2}{*}{ Design Metric } & \multicolumn{4}{|c|}{ Delay Budgeting Algorithms } & \multirow{2}{*}{$\begin{array}{c}\text { Variation among } \\
\text { Budgeting policies }(\%)\end{array}$} & \multirow{2}{*}{$\begin{array}{c}\text { Delay Budgeting } \\
\text { vs. no Budgeting (\%) }\end{array}$} \\
\hline & & No-budget & Max & Max-fair $(10 \%)$ & Max-fair $(25 \%)$ & & \\
\hline \multirow{4}{*}{ DFG0 } & LUT count & 1790 & $\overline{1342}$ & 1158 & 101478 & 27.63 & 35.30 \\
\hline & Slice count & 1232 & 1008 & 920 & 1104 & 8.73 & 33.91 \\
\hline & Total budget & - & 66 & 64 & 53 & 20.7 & - \\
\hline & Relaxed nodes & - & 8 & 8 & 8 & 0 & - \\
\hline \multirow{4}{*}{ DFG1 } & LUT count & 3856 & 2365 & 2168 & 2608 & 20.20 & 43.77 \\
\hline & Slice count & 2672 & 1930 & 1837 & 2090 & 13.77 & 31.72 \\
\hline & Total budget & - & 66 & 64 & 53 & 24.52 & - \\
\hline & Relaxed nodes & - & 13 & 18 & 18 & 38.46 & - \\
\hline \multirow{4}{*}{ DFG2 } & LUT count & 8658 & 5149 & 5102 & 4837 & 6.40 & 78.99 \\
\hline & Slice count & 6249 & 4525 & 4496 & 4355 & 3.90 & 43.49 \\
\hline & Total budget & - & 138 & 122 & 109 & 26.60 & - \\
\hline & Relaxed nodes & - & 28 & 38 & 40 & 42.85 & - \\
\hline \multirow{4}{*}{ DFG3 } & LUT count & 3168 & 2022 & 2075 & 2388 & 18.10 & 56.60 \\
\hline & Slice count & 2224 & 1657 & 1686 & 1864 & 12.49 & 34.21 \\
\hline & Total budget & - & 42 & 39 & 32 & 31.25 & - \\
\hline & Relaxed nodes & - & 11 & 12 & 12 & 9.09 & - \\
\hline \multirow{4}{*}{ Average } & LUT count & 4368.00 & 2719.5 & 2625.75 & 2827.75 & 18.08 & 53.66 \\
\hline & Slice count & 3094.25 & 2280.00 & 2234.75 & 2353.25 & 9.72 & 35.83 \\
\hline & Total budget & - & 78 & 72.25 & 61.75 & 25.76 & - \\
\hline & Relaxed nodes & - & 15 & 19 & 19.5 & 22.6 & - \\
\hline
\end{tabular}

Table 2: The quality of different delay budget assignment techniques.

\section{References}

[1] A. Kahng, S.Mantik, and I.L. Markov. "Min-Max Placement for Large-Scale Timing Optimization". In ACM International Symposium on Physical Design, pages 143-148, 2002.

[2] C. Chen, E. Bozorgzadeh, A. Srivastava and M. Sarrafzadeh. "Budget Management with Applications". Algorithmica, 34(3):261-275, July 2002.

[3] C. Chen, X. Yang, M. Sarrafzadeh. "Potential Slack: An Effective Metric of Combinational Circuit Performance". In ACM/IEEE International Conference on Computer-Aided Design, pages 198-201, 2000.

[4] C. Kuo and A. C.H. Wu. "Delay Budgeting for a Timing-Closure-Design Method". In ACM/IEEE International Conference on Computer-Aided Design, pages 202-207, 2000.

[5] C. Lee, M. Potkonjak, and W.H. Mangione-Smith. "MediaBench: A Tool for Evaluating and Synthesizing Multimedia and Communications Systems". In International Symposium on Microarchitecture, 1997.

[6] C. Yeh and M. Marek-Sadowska. "Delay Budgeting in Sequential Circuit with Application on FPGA Placement". In ACM/IEEE Design Automation Conference, 2003.

[7] D. Kagaris and S. Tragoudas. "Maximum Independent Sets on Transitive Graphs and Their Applications in Testing and CAD". In IEEE/ACM International Conference on Computer-Aided Design, pages 736-740, 1997.

[8] G.B. Dantzig. Linear Programming and Extensions. Princeton University Press, 1963.

[9] E. Boros, P. Hammer and R. Shamir. "A Polynomial Algorithm for Balancing Acyclic Data Flow Graphs". IEEE Trans. Computers, 41(11):1380-1385, 1992.

[10] E. Boros, P. Hammer, M. Hartmann and R. Shamir. "Balancing Problems in Acyclic Networks". Discrete Applied Math., 49(1-3):77-93, 1994.

[11] E. Bozorgzadeh, S. Ghiasi, A. Takahashi and M. Sarrafzadeh. "Optimal Integer Delay Budgeting on Directed Acyclic Graphs". In Design Automation Conference, June 2003.

[12] E. Felt, E. Charbon , E. Malavasi and A. Sangiovanni-Vincentelli. "An Efficient Methodology for Symbolic Compaction of Analog IC's with Multiple Symmetry Constraints". In Conference on European Design Automation, November 1992.

[13] H.R. Lin and T. Hwang. "Power Reduction by Gate Sizing with Path-Oriented Slack Calculation". In IEEE Asia and South Pacific Design Automation Conference (ASPDAC), pages 7-12, 1995.

[14] J. Luo and N. Jha. "Battery-Aware Static Scheduling for Distributed Real-Time Embedded Systems". In IEEE/ACM Design Automation Conference, 2001.

[15] J.F. Lee and D.T. Tang. "VLSI Layout Compaction with Grid and
Mixed Constraints". IEEE Transactions on Computer-Aided Design of Integrated Circuits and Systems, 6(5), September 1987.

[16] M. Sarrafzadeh, D. Knol and G.E. Tellez. "Unification of Budgeting and Placement". In ACM/IEEE Design Automation Conference, June 1997.

[17] M.D. Smith, and G. Holloway. "An introduction to machine SUIF and its portable libraries for analysis and optimization". Technical report, Division of Engineering and Applied Sciences, Harvard University, 2002.

[18] M.Sarrafzadeh, D.A. Knol and G.E. Tellez. "A Delay Budgeting Algorithm Ensuring Maximum Flexibility in Placement". IEEE Transactions on Computer-Aided Design of Integrated Circuits and Systems, pages 1332-1341, 1997.

[19] M.W. Hall, J.M. Anderson, S.P. Amarasinghe, B.R. Murphy, L. Shih-Wei, E. Bugnion, and M.S. Lam. "Maximizing Multiprocessor Performance with the SUIF Compiler". Computer, 29(12):84-89, 1996

[20] O. Coudert. "Timing and Design Closure in Physical Design Flows". In IEEE International Symposium on Quality Electronic Design, 2002.

[21] P. Girard, C. Landrault, S. Pravossoudovitch and D. Severac. ”A Gate Resizing Technique for High Reduction in Power Consumption". In International Symposium on Low Power Electronics and Design, pages 281-286, 1997.

[22] T. Magnanti R. Ahuja and J. Orlin. Network Flows: Theory, Algorithms, and Applications. Prentice Hall, 1993.

[23] R. Nair, C. Berman, P. Hauge and E. Yoffa. "Generation of Performance Constraints for Layout". IEEE Transactions on Computer-Aided Design of Integrated Circuits and Systems, 8:860-874, August 1989.

[24] S. Bakshi and D. Gajski. "Component Selection for High-Performance Pipelines". IEEE Transactions on Very Large Scale Integrated Systems, 4(2):181-194, 1996.

[25] R. Rivest T. Cormen, C. Leiserson. An introduction to algorithms. MIT Press, 1990.

[26] T. Kong. "A Novel Net Weighting Algorithm for Timing-Driven Placement". In ACM/IEEE International Conference on Computer-Aided Design, 2002.

[27] W. Zhang, N. Vijaykrishnan, M. Kandemir, M.J. Irwin, D. Duarte, and Y.Tsai. "Exploiting VLIW Schedule Slacks for Dynamic and Leakage Energy Reduction”. In ACM/IEEE International Symposium on Microarchitecture, 2001.

[28] Y. Liao and C. K. Wong. "An Algorithm to Compact a VLSI Symbolic Layout with Mixed Constraints". IEEE Transactions on Computer-Aided Design of Integrated Circuits and Systems, 2(2), April 1983. 